\title{
Study on the Correlation between Serum 25-Hydroxyvitamin D3 Level and Obesity
}

\author{
Weijuan Song", Ting Cui", Qiuxia Ge, Ruixia Yang* \\ Department of Laboratory Medicine, The First Affiliated Hospital of Nanjing Medical University, \\ National Key Clinical Department of Laboratory Medicine, Nanjing, China \\ "These authors contributed equally to this work. \\ *Corresponding author: welcomeyrx@163.com
}

Received May 15, 2019; Revised June 22, 2019; Accepted July 03, 2019

\begin{abstract}
Objective: To investigate the relationship between serum 25- hydroxyvitamin D3 [25-(OH)D3] and its related metabolic indexes in the obese. Methods: 239 obese patients, including 109 males and 130 females, were collected from the weight-loss clinic of Jiangsu People's Hospital from December 2016 to March 2018, and 221 physical examination samples, including 99 males and 122 females, were collected from physical examination Center of Jiangsu People's Hospital at the same time. The levels of 25-(OH)D3 were measured and their relationship with obesity, glycometabolism and lipid metabolism was analyzed. Results: BMI of males was significantly higher than that of females $(\mathrm{p}<0.05)$. There was no significant difference in the level of 25- $(\mathrm{OH}) \mathrm{D} 3$ between men and women. With the increase of BMI, the level of 25-(OH)D3 showed a downward trend, with a significant difference $\left(\chi^{2}=10.066, \mathrm{P}<0.05\right)$. The prevalence rate of simple obesity in 25-(OH)D3 more insufficient group was significantly lower than that in 25-(OH)D3 insufficient group $\left(\chi^{2}=6.121, \mathrm{P}<0.05\right)$. Although the prevalence rate of hypertension, type 2 diabetes mellitus, hyperuricemia and hyperinsulinemia in the 25-(OH)D3 more insufficient group was higher than that in the 25-(OH)D3 insufficient group, there was no significant difference. Pearson correlation analysis showed that 25-(OH)D3 was negatively correlated with BMI ( $r=-0.197)$, C-peptide (C-P) $(r=-0.134)$, and positively correlated with age $(\mathrm{r}=0.168)$. Taking $25-(\mathrm{OH}) \mathrm{D} 3$ as dependent variable and other indexes as independent variables, multivariate linear regression analysis was performed. Age, BMI, total cholesterol (TC), low density lipoprotein cholesterol (LDL-C) were independently correlated with 25-(OH)D3 ( beta values were 0.157, -0.164, 0.513 and0.473 respectively). Conclusion: Obesity and related metabolic abnormalities are closely related to the decrease of 25-(OH)D3. The level of 25-(OH)D3 usually decreased in obese people, and the decrease of 25-(OH)D3 will lead to metabolic abnormalities such as obesity and hypertension.
\end{abstract}

Keywords: 25- hydroxyvitamin D3, obesity, metabolic abnormalities

Cite This Article: Weijuan Song, Ting Cui, Qiuxia Ge, and Ruixia Yang, "Study on the Correlation between Serum 25-Hydroxyvitamin D3 Level and Obesity.” American Journal of Medicine Studies, vol. 7, no. 1 (2019): 1-6. doi: 10.12691/ajms-7-1-1.

\section{Introduction}

Obesity is a chronic metabolic disease caused by many factors. It is characterized by abnormal increase in body fat percentage due to the increase in the volume and number of adipocytes in the body and excessive accumulation of fat in some parts of the body. With the improvement of people's living standard and the change of dietary structure, the incidence of obesity is increasing year by year, which has become the root cause of most public health problems. Vitamin D is a fat-soluble vitamin, which can regulate calcium and phosphorus metabolism. Many studies have shown that vitamin D plays an important role in immunity, tumors, cardiovascular diseases and internal diseases [1-6]. A few studies abroad have shown that obese people are prone to vitamin D deficiency [7,8]. At present, there are few studies on the correlation between obesity and vitamin D in Chinese population. This paper retrospectively analyzed the relationship between serum levels of 25-hydroxyvitamin D3 [25-(OH)D3] and body mass index (BMI), glycometabolism and lipid metabolism of obesity patients in weight loss clinic of Jiangsu People's Hospital from December 2016 to March 2018 To explore whether the change of vitamin $\mathrm{D}$ level will increase the risk of obesity and related metabolic abnormalities, and provide scientific basis for clinical intervention of obesity.

\section{Materials and Methods}

\subsection{Research Object}

239 obese patients from December 2016 to March 2018 in the weight-loss clinic of Jiangsu People's Hospital were collected, including 109 males, and 130 females, 
with an average age of $30.13 \pm 8.77$ years, 221 physical examination samples from physical examination Center of Jiangsu People's Hospital were collected at the same time, including 99 males and 122 females, with an average age of $30.94 \pm 9.42$ years. Inclusion criteria: 1 . Physical health on weekdays, no history of rickets, arthralgia, malnutrition, genetic metabolic diseases and other chronic diseases, 2. No drugs affecting vitamin D absorption or bone metabolism were taken, 3 . No thyroid dysfunction, renal dysfunction and other diseases affecting vitamin D level, 4. Normal sunshine exposure, 5 . No pregnancy, cancer, ascites, severe liver, kidney dysfunction and severe cardiovascular and cerebrovascular diseases. All subjects tested included height, weight, blood pressure, total cholesterol (TC), low density lipoprotein cholesterol (LDL-C), C-peptide (C-P), 25-hydroxyvitamin D3 [25-(OH)D3], triglyceride (TG), high density lipoprotein cholesterol (HDL-C), uric acid (UA), fasting blood glucose (FBG), insulin (INS). This study meets the ethical requirements of the Helsinki Declaration. All participants are aware of the process of this study and volunteer to participate in this study.

\subsection{Research Method}

\subsubsection{Height and Weight Measurement Method}

When measuring height and weight, we should take off shoes, hats and wear thin clothes and pants, after excretion of feces and urine in the morning. The subjects were standing on the floor of the instrument for measuring height and weight in an "upright" position, with their heels, sacrum and shoulder blades close to the instrument 's posts. The surveyor can stand on the left and right sides of the measured person, Adjust the head to the lowest point of the upper ear margin and the infraorbital margin of eye, and then move the horizontal plate of the instrument for measuring height and weight to the top of the measured person's head, reading height and weight. The unit of height is $\mathrm{cm}$ and the unit of weight is $\mathrm{kg}$. BMI = body weight (kg) / height (m) ${ }^{2}$.

\subsubsection{The Method of Sitting Blood Pressure Measurement}

Mercury column sphygmomanometer, let the measured person rest quietly for 15 minutes before measuring, and determine that there is no smoking and vigorous activity within at least half an hour; the arm of the examined person should be at the same height as the heart position (the level of the fourth rib when sitting), The cuff should be laid flat, the lower edge of the cuff should be 2-3 $\mathrm{cm}$ away from the cubital fossa, and the tightness should be inserted into two fingers. When pressurizing, the mercury column should be raised $20-30 \mathrm{mmHg}$ above the base systolic pressure, the deflation should be slow, the systolic pressure should be based on the first sound, and the diastolic pressure should be based on the sound disappearance or sound change. Continuous measurement 3 times (30 seconds interval each time), take the average value of measurement.

\subsubsection{Detection of Blood Parameters}

Fasting (fasting 12-14h) venous blood 3ml was collected from the subjects in vacuum inert separation gel conduit.
After centrifugation for 5 minutes at 3000r/min (centrifugal radius $17.49 \mathrm{~cm}$ ), the upper serum was collected. 25-(OH)D3, INS and CP were detected by Roche Cobase 602 electrochemiluminescent immunoassay instrument and its matching reagents. TC, TG, LDL-C, HDL-C, UA and FBG were detected by Beckman AU5800 automatic biochemical analyzer and reagents produced by Shanghai Haiershi Diagnostic Products. All the tests were strictly operated according to the instrument and reagent instructions, and each batch of tests were tested for low value and high value quality control.

\subsubsection{Diagnos Tic Criteria for Vitamin D Deficiency [9]}

According to international classification criteria for 25-(OH)D3 deficiency, 25-(OH) D3 is sufficient $\geq 75 \mathrm{nmol} / \mathrm{L}$, $25-(\mathrm{OH}) \mathrm{D} 3$ is insufficient to $50-74 \mathrm{nmol} / \mathrm{L}, 25-(\mathrm{OH}) \mathrm{D} 3$ is more insufficient $\leq 49 \mathrm{nmol} / \mathrm{L})$.

\subsubsection{Statistical Method}

SPSS13.0 software was used for statistical analysis of the data. Because of skewness and kurtosis of the distributions, datas were described by median values with interquartile ranges (25th to 75th). Univariate analyses included one-way ANOVA, Kruskal-Wallis test, and $\chi 2$ test., Pearson correlation analysis was used for correlation analysis, and multivariate linear regression was used for multivariate analysis. The difference was statistically significant with $\mathrm{P}<0.05$.

\section{Results}

\subsection{General Characteristics of Research Population}

There was no significant difference in age between the two groups $\left.\chi^{2}=0.832, \mathrm{P}=0.362>0.05\right)$. Systolic blood pressure, diastolic blood pressure, BMI, TC, TG, UA, FBG, INS, C-P, insulin resistance index in obese group were significantly higher than those in control group $(\mathrm{P}<0.05)$; HDL, 25- $(\mathrm{OH})$ D3 in obese group were significantly lower than those in control group $(\mathrm{P}<0.05)$, the difference was significant $(\mathrm{P}<0.05)$, as shown in Table 1.

In the obese group, including 109 males, aged 13-61 years and 130 females, aged 20-54 years. The average BMI index of males was significantly higher than that of females $(\chi 2=10.007, \quad \mathrm{P}=0.002)$, and there was no significant difference in the level of 25-(OH)D3 between males and females, as shown in Table 2.

\subsection{Comparison of 25-(OH)D3 levels in different obesity degrees}

The quartiles of BMI in the research population were $\mathrm{P} 25=33.13 \mathrm{~kg} / \mathrm{m}^{2}, \mathrm{P} 50=37.19 \mathrm{~kg} / \mathrm{m}^{2}$ and $\mathrm{P} 75=42.15 \mathrm{~kg} / \mathrm{m}^{2}$, respectively. The research population was divided into four groups according to BMI quartile spacing: group A: BMI $<33.13 \mathrm{~kg} / \mathrm{m}^{2}$, group B: $33.13 \mathrm{~kg} / \mathrm{m}^{2} \leq \mathrm{BMI}<37.19 \mathrm{~kg} / \mathrm{m}^{2}$, group C: $37.19 \mathrm{~kg} / \mathrm{m}^{2} \leq \mathrm{BMI}<42.15 \mathrm{~kg} / \mathrm{m}^{2}$, group D: BMI $\geq 42.15 \mathrm{~kg} / \mathrm{m}^{2}$. 
Table 1. Clinical characteristics of obesity group and control group

\begin{tabular}{lcccc}
\hline \multirow{2}{*}{ Parameter } & Obesity group & Control group & \multirow{2}{*}{$\chi^{2}$} & \multirow{2}{*}{$P$} \\
\cline { 2 - 3 } & $\mathbf{n}=\mathbf{2 3 9}$ & $30.00(14.00-61.00)$ & 0.832 & 0.362 \\
Age (years) & $28.00(13.00-61.00)$ & $118.00(91-139)$ & 96.62 & 0.000 \\
SBP (mmHg) & $131.00(90.00-219.00)$ & $80.00(65-89)$ & 4.28 & 0.039 \\
DBP (mmHg) & $82.00(42.00-151.00)$ & $21.30(18.60-23.60)$ & 343.79 & 0.000 \\
BMI (kg/m²) & $37.19(24.50-67.00)$ & $4.18(1.85-8.34)$ & 23.76 & 0.000 \\
TC (mmol/L) & $4.66(2.11-9.75)$ & $1.34(0.46-12.62)$ & 2.34 & 0.020 \\
TG (mmol/L) & $1.65(0.48-19.09)$ & $3.25(1.82-5.86)$ & 0.037 & 0.848 \\
LDL (mmol/L) & $3.24(0.91-6.85)$ & $1.32(0.6-2.23)$ & 109.21 & 0.000 \\
HDL (mmol/L) & $0.99(0.51-1.94)$ & $312.80(124.00-559.00)$ & 113.97 & 0.000 \\
UA (umol/L) & $411.00(176.00-725.00)$ & $5.34(1.85-15.50)$ & 3.95 & 0.047 \\
FBG (mmol/L) & $5.56(3.44-16.79)$ & $68.40(14.70-231.00)$ & 180.80 & 0.000 \\
INS (pmol/L) & $181.60(6.00-4534.00)$ & $750.00(623.00-2223.00)$ & 169.88 & 0.000 \\
C-P (pmol/L) & $1448.00(161.70-5117.00)$ & $55.10(23.00-122.80)$ & 96.93 & 0.000 \\
25-(OH)D3(nmol/L) & $38.70(11.59-111.50)$ & $12.82(3.86-22.80)$ & 235.88 & 0.000 \\
Insulin Resistance Index & $32.66(1.74-270.04)$ & & \\
\hline
\end{tabular}

Note: SBP: systolic pressure, DBP: diastolic pressure, BMI: body mass index, TC: total cholesterol, TG: triglyceride, LDL: low density lipoprotein cholesterol, HDL: high density lipoprotein cholesterol, UA: uric acid, FBG: fasting blood sugar, INS: insulin,C-P: C-peptide, 25-(OH) D3: 25hydroxyvitamin D3.

Table 2. Clinical characteristics of obesity population

\begin{tabular}{lcccc}
\hline Parameter & Male & Female & $\chi^{2}$ & $\mathrm{P}$ \\
& $\mathrm{n}=109$ & $30.00(20.00-54.00)$ & 1.992 & 0.158 \\
Age (years) & $29.00(13.00-61.00)$ & $126.50(90.00-219.00)$ & 25.569 & 0.000 \\
SBP (mmHg) & $136.00(101.00-190.00)$ & $80.00(42.00-151.00)$ & 16.018 & 0.000 \\
DBP (mmHg) & $85.00(60.00-134.00)$ & $36.16(18.37-67.00)$ & 10.007 & 0.002 \\
BMI $\left(\mathrm{kg} / \mathrm{m}^{2}\right)$ & $39.24(24.50-62.46)$ & $4.74(2.69-9.75)$ & 0.249 & 0.618 \\
TC(mmol/L) & $4.63(2.11-8.14)$ & $1.53(0.50-19.09)$ & 5.448 & 0.020 \\
TG(mmol/L) & $1.71(0.38-8.06)$ & $3.12(1.50-6.85)$ & 5.352 & 0.021 \\
LDL(mmol/L) & $3.34(0.91-5.45)$ & $1.02(0.58-1.94)$ & 16.684 & 0.000 \\
HDL(mmol/L) & $0.94(0.51-1.47)$ & $390.00(176.00-637.00)$ & 18.813 & 0.000 \\
UA (umol/L) & $449.00(224.00-725.00)$ & $5.40(3.57-16.12)$ & 4.093 & 0.043 \\
FBG(mmol/L) & $5.90(3.44-16.79)$ & $168.60(8.30-1299.00)$ & 2.460 & 0.117 \\
INS(pmol/L) & $193.40(6.00-4534.00)$ & $1359.00(161.70-3704.00)$ & 5.562 & 0.018 \\
C-P (pmol/L) & $1494.00(249.80-5117.00)$ & $38.90(14.30-111.50)$ & 0.856 & 0.355 \\
25-(OH)D3 (nmol/L) & $38.60(11.59-104.03)$ & $29.53(1.80-200.70)$ & 0.697 & 0.404 \\
Insulin Resistance Index & $33.10(1.74-270.04)$ & & & \\
\hline
\end{tabular}

Note: SBP: systolic pressure, DBP: diastolic pressure, BMI: body mass index, TC: total cholesterol, TG: triglyceride, LDL: low density lipoprotein cholesterol, HDL: high density lipoprotein cholesterol, UA: uric acid, FBG: fasting blood sugar, INS: insulin C-P: C-peptide, 25-(OH) D3: 25hydroxyvitamin D3.

Trend test analysis showed that with the increase of BMI, the level of 25 - $(\mathrm{OH})$ D3 showed a downward trend, with a significant difference $(\chi 2=10.066, \mathrm{P}<0.05)$, as shown in Table 3. Linear trend lines show that 25-(OH)D3 decreases by 0.41 units for each additional unit of BMI without considering other factors (see Figure 1). With the increase of obesity, the rate of 25-(OH)D3 deficiency increased gradually. The difference was statistically significant $\left(\chi^{2}=14.576, P<0.05\right)$, as shown in Table 4.

Table 3. Analysis of 25-(OH)D3 levels in different obesity degrees

\begin{tabular}{ccc}
\hline Group & Number of cases & $25-(\mathrm{OH}) \mathrm{D} 3(\mathrm{nmol} / \mathrm{L})$ \\
\hline Group A & 59 & $45.70 \pm 15.12$ \\
Group B & 59 & $41.18 \pm 14.26$ \\
Group C & 58 & $39.37 \pm 18.95$ \\
Group D & 63 & $36.16 \pm 18.30$ \\
\hline
\end{tabular}

Note: Group A: BMI $<33.13 \mathrm{~kg} / \mathrm{m}^{2}$, Group B: $33.13 \mathrm{~kg} / \mathrm{m}^{2} \leq$ BMI $<$ $37.19 \mathrm{~kg} / \mathrm{m}^{2}$, Group C: $37.19 \mathrm{~kg} / \mathrm{m}^{2} \leq \mathrm{BMI}<42.15 \mathrm{~kg} / \mathrm{m}^{2}$ and Group D: $\mathrm{BMI} \geq 42.15 \mathrm{~kg} / \mathrm{m}^{2}$, the same below.

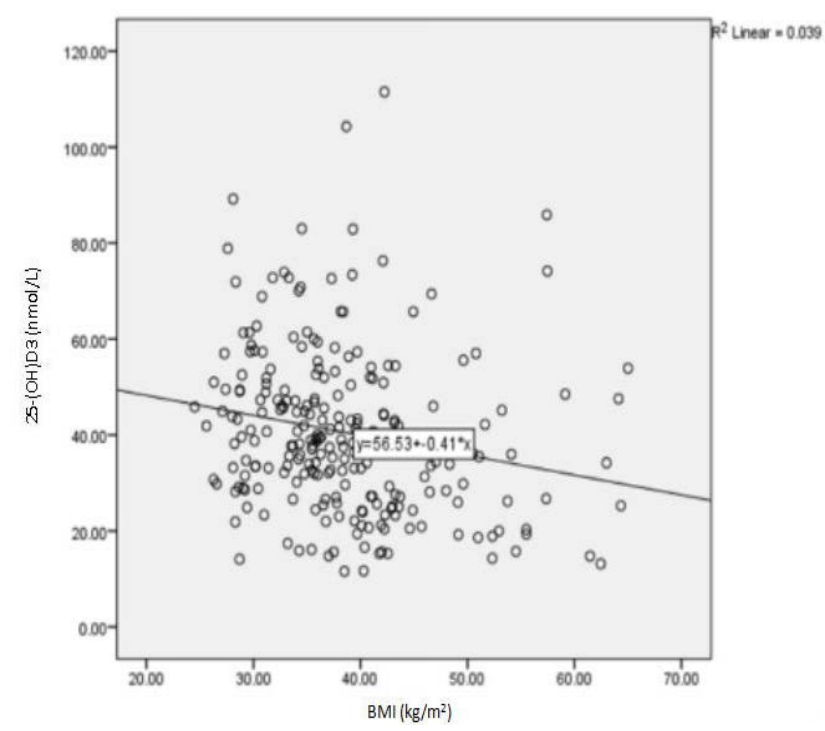

Figure 1. Linear trend scatter plots of BMI and 25-(OH)D3 
Table 4. Comparison of prevalence of vitamin $\mathrm{D}$ deficiency or more deficiency in different degrees of obesity(\%)

\begin{tabular}{|c|c|c|c|c|}
\hline Group & $\begin{array}{c}\text { Group A } \\
n=59\end{array}$ & $\begin{array}{c}\text { Group B } \\
n=59\end{array}$ & $\begin{array}{c}\text { Group C } \\
n=58\end{array}$ & $\begin{array}{c}\text { Group D } \\
n=63\end{array}$ \\
\hline 25-(OH)D3 more insufficient & $36(61.02 \%)$ & $46(77.97 \%)$ & $49(84.48 \%)$ & $55(87.30 \%)$ \\
\hline 25-(OH)D3 insufficient & $21(35.59 \%)$ & $12(20.34 \%)$ & $8(13.79 \%)$ & $7(11.11 \%)$ \\
\hline 25-(OH)D3 sufficient & 2 (3.39\%) & $1(1.69 \%)$ & $1(1.72 \%)$ & $1(1.59 \%)$ \\
\hline
\end{tabular}

Table 5. Prevalence comparison of 25(OH)D3 insufficient group and 25(OH)D3 more insufficient group(\%)

\begin{tabular}{|c|c|c|c|c|}
\hline Disease types & $\begin{array}{l}\text { 25(OH)D3 insufficient group } \\
\qquad n=50\end{array}$ & $\begin{array}{l}\text { 25-(OH)D3 more insufficient group } \\
\qquad \mathrm{n}=181\end{array}$ & $\chi^{2}$ & $\mathrm{P}$ \\
\hline Obesity & $23(46.00 \%)$ & $50(27.62 \%)$ & 6.121 & 0.013 \\
\hline Obesity + Hypertension & $2(4.00 \%)$ & $9(4.97 \%)$ & 0.082 & 0.775 \\
\hline Obesity + Type 2 diabetes mellitus & $6(12.00 \%)$ & $31(17.13 \%)$ & 0.766 & 0.382 \\
\hline Obesity + Hyperuricemia & $4(8.00 \%)$ & $21(11.60 \%)$ & 0.527 & 0.468 \\
\hline Obesity + Hyperinsulinemia & $3(6.00 \%)$ & $16(8.84 \%)$ & 0.419 & 0.518 \\
\hline Obesity + Type 2 diabetes mellitus + Hyperuricemia & $1(2.00 \%)$ & $5(2.76 \%)$ & 0.090 & 0.764 \\
\hline Obesity + Type 2 diabetes mellitus + Hypertension & $5(10.00 \%)$ & $22(12.15 \%)$ & 0.176 & 0.675 \\
\hline Obesity + Hyperinsulinemia + Hyperuricemia & $6(12.00 \%)$ & $27(14.92 \%)$ & 0.272 & 0.602 \\
\hline
\end{tabular}

Table 6. Relevance of 25-(OH)D3 with Indicators

\begin{tabular}{|c|c|c|c|c|c|c|}
\hline \multirow{2}{*}{ Parameter } & \multicolumn{2}{|c|}{ Bivariate correlation } & \multicolumn{4}{|c|}{ Multiple regression analysis } \\
\hline & $\mathrm{r}$ & $\mathrm{p}$ & $\beta$ & $\mathrm{t}$ & $\mathrm{p}$ & $95 \% \mathrm{CI}$ \\
\hline Age (years) & 0.168 & 0.009 & 0.157 & 2.188 & 0.030 & $0.030-0.581$ \\
\hline SBP (mmHg) & -0.033 & 0.617 & -0.041 & -0.375 & 0.708 & $-0.266-0.181$ \\
\hline DBP (mmHg) & 0.000 & 1.000 & 0.087 & 0.810 & 0.419 & $-0.158-0.379$ \\
\hline $\mathrm{BMI}\left(\mathrm{kg} / \mathrm{m}^{2}\right)$ & -0.197 & 0.002 & -0.164 & -2.237 & 0.026 & $-0.649--0.041$ \\
\hline $\mathrm{TC}(\mathrm{mmol} / \mathrm{L})$ & 0.056 & 0.392 & 0.513 & 2.547 & 0.012 & $1.896-14.863$ \\
\hline TG(mmol/L) & 0.008 & 0.897 & -0.152 & -1.765 & 0.079 & $-2.811-0.154$ \\
\hline LDL(mmol/L) & -0.035 & 0.587 & -0.473 & -2.646 & 0.009 & $-17.132--2.507$ \\
\hline $\mathrm{HDL}(\mathrm{mmol} / \mathrm{L})$ & 0.093 & 0.151 & -0.043 & -0.478 & 0.633 & $-15.773-9.614$ \\
\hline $\mathrm{UA}(\mu \mathrm{mol} / \mathrm{L})$ & -0.028 & 0.662 & 0.089 & 1.157 & 0.249 & $-0.010-0.038$ \\
\hline $\mathrm{FBG}(\mathrm{mmol} / \mathrm{L})$ & 0.069 & 0.289 & 0.069 & 0.749 & 0.454 & $-0.775-1.725$ \\
\hline INS(pmol/L) & -0.071 & 0.276 & -0.078 & -0.286 & 0.775 & $-0.032-0.024$ \\
\hline C-P(pmol/L) & -0.134 & 0.038 & -0.064 & -0.522 & 0.602 & $-0.008-0.004$ \\
\hline Insulin Resistance Index & -0.103 & 0.113 & 0.058 & 0.184 & 0.854 & $-0.236-0.284$ \\
\hline
\end{tabular}

\subsection{Prevalence Comparison of 25(OH)D3 Insufficient Group and 25(OH)D3 More Insufficient Group}

The prevalence of simple obesity in 25-(OH)D3 more insufficient group was significantly lower than that in $25-(\mathrm{OH}) \mathrm{D} 3$ insufficient group $(\mathrm{P}<0.05)$. The prevalence of hypertension, type 2 diabetes mellitus, hyperuricemia and hyperinsulinemia in 25-(OH)D3 more insufficient group was higher than that that in 25-(OH)D3 insufficient group there was no significant difference $(P>005)$, as shown in Table 5.

\subsection{Relevance of $25-(\mathrm{OH}) \mathrm{D} 3$ with Indicators}

Pearson correlation analysis showed that 25-(OH)D3 was negatively correlated with BMI, C-peptide, and positively correlated with age, with statistical significance. Taking 25-(OH)D3 as dependent variable and other indexes as independent variables, multivariate linear regression analysis was performed. Age, BMI, TC, LDL-C were independently correlated with 25-(OH)D3, beta values were $0.157,-0.164,0.513$ and- 0.473 . as shown in Table 6.

\section{Discussion}

Vitamin D is a lipid-soluble steroid derivative with biological activity. Its main source is the conversion of 7-dehydrocholesterol from skin to vitamin D3 by photochemistry of ultraviolet rays in the sun. At the same time, the transcription and translation of vitamin D sensitive genes are enhanced, and then vitamin D3 is hydroxylated into25-(OH)D3 in the liver, which is the main form of vitamin D in the blood circulation [10]. In recent years, there has been a lot of progress in the research of vitamin $\mathrm{D}$ at home and abroad. Viljakainen et al. have shown that vitamin $\mathrm{D}$ is an important hormone precursor, which can regulate $3 \%$ of the human genome, and the decrease of vitamin D content will have many effects on the body [11] Vitamin D plays a role in the occurrence and development of tumors, autoimmune diseases, metabolic diseases and endocrine diseases. In recent years, the correlation between vitamin $\mathrm{D}$ and obesity has attracted considerable attention in the world, but few studies have been conducted in China. This study explored the correlation between serum 25-(OH)D3 and obesity, and provided scientific basis for preventing obese and the occurrence and development of metabolic syndrome in obese. 
Some studies have confirmed that serum 25-(OH)D3 is negatively correlated with BMI [12]. Filippou et al. have shown that serum 25-(OH)D3 is closely related to obesity and insulin resistance [13]. The relationship between serum 25-(OH)D3 level and body mass index, glycometabolism and lipid metabolism in 239 obese patients was analyzed in our study. The results showed that the level of $25-(\mathrm{OH}) \mathrm{D} 3$ decreased with the increase of BMI, and the prevalence of vitamin $\mathrm{D}$ deficiency increased with the increase of obesity. This further confirms that vitamin D plays an important regulatory role in the development of obesity. Some scholars believe that the exact mechanism of the effect of vitamin D deficiency on obesity is that vitamin $\mathrm{D}$ deficiency can lead to increased levels of parathyroidhormone (PTH), calcium influx in adipocytes, activation of genes related to calcium metabolism and increase the activity of enzymes in lipid synthesis. Thus, fat accumulation in adipocytes increases weight. 1,25-(OH)2D3 can inhibit adipogenesis independently of PTH and plays an important role in regulating adipocyte metabolism [14].

Decreased vitamin D levels not only lead to obesity, but also increase the risk of metabolic abnormalities. The results showed that the prevalence of hypertension, type 2 diabetes mellitus, hyperuricemia and hyperinsulinemia increased significantly in 25-(OH)D3 deficiency patients. At present, vitamin D is thought to affect blood pressure by regulating renin angiotensin system (RAS) [15]. Studies have shown that 25-(OH)D3 can be expressed in pancreatic $\beta$ cells, which helps to maintain intracellular and extracellular calcium concentration, while insulin secretion is a calcium-dependent process [16,17]. In addition, there are vitamin $\mathrm{D}$ (VD) receptors on islet $\beta$ cells. VD promotes insulin synthesis and secretion by binding vitamin D binding protein to VD receptor, And then affect the blood sugar concentration. Therefore, obese patients should dynamically monitor the level of $25-(\mathrm{OH}) \mathrm{D} 3$ in the process of weight loss and supplement vitamin $\mathrm{D}$ when necessary, so as to reduce the occurrence and development of metabolic diseases.

In this study, the correlation between 25-(OH)D3 level and glycometabolism, lipid metabolism in 239 obese patients was analyzed. The results showed that 25-(OH)D3 was negatively correlated with BMI and C-peptide. Taking 25-(OH)D3 as dependent variable and other indexes as independent variables, multivariate linear regression analysis was performed. Age, BMI, TC and LDL-C were independently correlated with 25-(OH)D3. It is demonstrated again that vitamin $\mathrm{D}$ plays an important role in the metabolism of sugar and lipid. Gonzalez et al.believe that vitamin D can promote the expression of cytochrome P450 Superfamily A1 (CYPA1) through the activation of vitamin D receptors. Thus, it can regulate the metabolism of cholesterol [18].

In conclusion, vitamin D is closely related to obesity and related metabolic syndrome. Obesity leads to the decrease of vitamin D level. Conversely, decreased vitamin $\mathrm{D}$ increases the risk of obesity and related metabolic abnormalities. Obese people should pay more attention to outdoor activities, receive enough sunlight, and eat vitamin D-rich foods to correct their own vitamin $\mathrm{D}$ deficiency. When necessary, vitamin $\mathrm{D}$ nutritional fortifiers should be supplemented properly to prevent and treat various metabolic syndrome caused by vitamin D deficiency. However, it is still worth exploring whether vitamin D supplements can prevent obesity and related metabolic syndrome, which may become a hot topic in future studies.

\section{Acknowledgements}

We are grateful to the technical support from National Key Clinical Department of Laboratory Medicine of Jiangsu Province Hospital.

\section{Disclosure of conflict of interest}

None.

\section{References}

[1] Vanherwegen AS, Gysemans C, Mathieu C, Regulation of Immune Function by Vitamin D and Its Use in Diseases of Immunity, Endocrinol Metab Clin North Am, 46(4). 1061-1094. Dec. 2017.

[2] Hossain MJ, Levinson A, George D, Canas J, Kumar S, and Balagopal PB, Vitamin D Status and Cardiovascular Risk in Obesity: Effect of Physical Activity in Nonvitamin D Supplemented Adolescents, Metab Syndr Relat Disord, 16(4). 197-203. May. 2018 .

[3] Nappi L, Ottaviano M, Rescigno P, Fazli L, Gleave ME, Damiano V, De Placido S and Palmieri G, Long term deficiency of vitamin D in germ cell testicular cancer survivors, Oncotarget, 20.9(30). 21078-21085. Apr.2018.

[4] Krysiak R, Szkróbka W, Okopień B, The Effect of Vitamin D on Thyroid Autoimmunity in Levothyroxine-Treated Women with Hashimoto's Thyroiditis and Normal Vitamin D Status, Exp Clin Endocrinol Diabetes, 125(4). 229-233. Apr.2017.

[5] Horas K, Maier G, Jakob F, Maus U, Kurth A, Jakuscheit A, Rudert M, and Holzapfel BM, High Prevalence of Vitamin D Deficiency in Patients with Bone Tumors, Cancer Invest, 35(8). 562-568. Sep.2017.

[6] Pilz S, Verheyen N, Grübler MR, Tomaschitz A and März W, Vitamin D and cardiovascular disease prevention, Nat Rev Cardiol, 13(7). 404-417. Jul. 2016.

[7] Walker GE, Follenzi A, Bruscaggin V, Manfredi M, Bellone S, Marengo E, Maiuri L, Prodam F,and Bona G, Fetuin B links vitamin $\mathrm{D}$ deficiency and pediatric obesity: Direct negative regulation by vitamin D, J Steroid Biochem Mol Biol, 182.37-49. Sep. 2018.

[8] Greene-Finestone LS, Garriguet D, Brooks S, Langlois K, and Whiting SJ, Overweight and obesity are associated with lower vitamin D status in Canadian children and adolescents, Paediatr Child Health, 22(8). 438-444. Nov.2017.

[9] Cava RC, Javier AN, Vitamin D deficiency, N Engl J Med, 8.357(19). 1981-1982. Nov.2007.

[10] Walsh JS, Bowles S, Evans AL, Vitamin D in obesity, Curr Opin Endocrinol Diabetes Obes, 24(6). 389-394. Dec.2017.

[11] Viljakainen HT, Saarnio E, Hytinantti T, Miettinen M, Surcel H, Mäkitie O, Andersson S, Laitinen K, and Lamberg-Allardt C, Maternal vitamin D status determines bone variables in the newborn, J Clin Endocrinol Metab, 95(4). 1749-1757. Apr. 2010.

[12] Pourshahidi LK, Vitamin D and obesity: current perspectives and future directions, Proc Nutr Soc, 74(2). 115-124. May. 2015.

[13] Moschonis G, Androutsos O, Hulshof T, Dracopoulou M, Chrousos GP, and Manios Y, Vitamin D insufficiency is associated with insulin resistance independently of obesity in primary schoolchildren. The healthy growth study, Pediatr Diabetes, 19(5). 866-873. Aug. 2018.

[14] Khundmiri SJ, Murray RD, Lederer E, PTH and Vitamin D, Compr Physiol, 6(2): 561-601. Mar. 2016. 
[15] Shu L, Huang K, Effect of vitamin D supplementation on blood pressure parameters in patients with vitamin D deficiency: a systematic review and meta-analysis, J Am Soc Hypertens, 12(7). 488-496. Jul. 2018.

[16] Lips P, Eekhoff M, van Schoor N, Oosterwerff M, de Jongh R, Krul-Poel Y, and Simsek S, Vitamin D and type 2 diabetes, J Steroid Biochem Mol Biol, 173. 280-285. Oct .2017.

[17] Sahebi R, Rezayi M, Emadzadeh M, Salehi M, Tayefi M, Parizadeh SM, Behboodi N, Rastgar-Moghadam A, Kharazmi
Khorassani J, Khorassani SK, Mohammadi A, Ferns GA, and Ghayour Mobarhan M, The effects of vitamin D supplementation on indices of glycemic control in Iranian diabetics: A systematic review and meta-analysis, Complement Ther Clin Pract, 34. 294-304. Feb. 2019.

[18] Gonzalez FJ, Moschetta A, Potential role of the vitamin D receptor in control of cholesterol levels, Gastroenterology, 146(4). 899-902. Apr. 2014. 